\title{
Kirchen und Christen im demokratischen Verfassungsstaat
}

\author{
von Friedrich Wilhelm Graf
}

Trotz immer wieder beschworener Niedergangsszenarien sind die beiden großen deutschen Volkskirchen weiterhin bedeutende gesellschaftliche und auch politische Akteure: Mehr als 50 Mio. Mitglieder wissen um die seelsorgerischen wie sozialen, nicht zuletzt aber auch ethischen Dienstleistungen dieser auf vielen Ebenen mit dem Staatswesen verbundenen Organisationen. Beide mussten sich den erfolgreichen Umgang mit der bundesdeutschen freiheitlichen Demokratie erst in mühsamen Lernprozessen erarbeiten; gelegentlich tun sich ihre Würdenträger auch heute noch schwer damit, ihre interne Diskurshoheit nicht mit einem gesamtgesellschaftlichen Deutungs- und Lenkungsanspruch zu verwechseln. Dennoch motiviert der Glaube weiterhin viele Menschen zum gesellschaftlichen Engagement, nicht nur bei den großen Sozialholdings Diakonisches Werk und Caritas, sondern auch im Rahmen kleinerer, meist lokaler Projekte. Eine gelungene Verbindung von demokratisch verfasstem Staat und institutionalisiertem Glauben bedarf daher eines gemeinsamen Diskursverständnisses, das im Sinne der Tradition des liberalen Kulturprotestantismus die rational vorgetragene Haltung der jeweils anderen (und des freien Individuums) ernst nimmt und davon absieht, Alleinvertretungsansprüche zu erheben.

Notwithstanding prophesised scenarios of organisational decline, both large German Volkskirchen remain important social as well as political actors: more than 50 million members know of the pastoral, social as well as ethical services provided by the 'people's churches', which still maintain strong links to the state on several levels. Both organisations had to go through difficult processes of learning how to deal successfully with (West) Germany's post-war democracy. Even today, some of their representatives find it difficult not to confuse their internal discursive dominance with an overarching authorisation to define the social and political agenda. Nevertheless, faith motivates many people to take on social responsibilities, be it in the large 'social holdings' Diakonisches Werk and Caritas or in small-scale projects at the local level. Any successful connection of democratic statehood and institutionalised religion, in the tradition of liberal cultural Protestantism, thus requires a common understanding of discursive practice that takes seriously the rational argument of the respective other side (or any free individual) and refrains from claiming exclusive moral, social, cultural and political authority. 


\section{Einleitung}

In der Bundesrepublik sind die beiden großen christlichen Volkskirchen höchst einflussreiche, mächtige Organisationen. Ihnen gehören ca. $60 \%$ der deutschen Bevölkerung an. Die Römisch-Katholische Kirche zählte 2005 rund 25,9 Millionen Mitglieder, und den evangelischen Landeskirchen gehörten damals 25,4 Millionen an. Hinzu kommen ca. 1 Million Christen in den verschiedenen orthodoxen Kirchen, von der Griechisch-Orthodoxen und der Serbisch-Orthodoxen Kirche bis hin zur Rumänisch-Orthodoxen und Russisch-Orthodoxen Kirche, die jeweils eigene administrative und seelsorgerliche Strukturen in Deutschland aufgebaut haben. Trotz aller religiösen Pluralisierung, wie sie insbesondere durch die Einwanderung von Menschen aus muslimisch geprägten Gesellschaften befördert wird, sowie der vielfältig sichtbaren Erosion der beiden etablierten Volkskirchen versteht sich die Mehrheit der in der Bundesrepublik lebenden Menschen mehr oder minder engagiert als Christen. Insoweit kann es nicht überraschen, dass den Kirchen und hier vor allem den beiden großen Volkskirchen im politischen System der Bundesrepublik vielfältige Machtchancen eingeräumt werden. Die beiden großen Kirchen sind, soziologisch gesehen, nun einmal die nach Mitgliederzahlen größten gesellschaftlichen Organisationen im Lande.

Ihr großer Einfluss wird insbesondere mit Blick auf den deutschen Sozialstaatskorporatismus deutlich: In der Sozialstaatskonstruktion spielen die eng mit den beiden Kirchen verknüpften Sozialholdings Diakonisches Werk und Caritas eine bestimmende Rolle. In den Tausenden von Sozialdienstleistungsunternehmen, Stiftungen, „Werken“ und „Diensten“, die in Caritas und Diakonischem Werk zusammengeschlossen sind, arbeiten jeweils mehr als 420.000 hauptberuflich tätige Mitarbeiter. Auch wenn sich Diakonie und Caritas eine relative Selbständigkeit gegenüber den Kirchenbürokratien bewahrt haben - eine von den Kirchenleitungen zunehmend bedrohte relative Autonomie! -, sind sie dank ihrer vielfältigen engen Verbindungen zu den Kirchen doch als Agenturen zu sehen, mit denen die Kirchen erheblichen gesellschaftlichen Einfluss ausüben. Nach ,dem Staat“ - Bund, Ländern und Kommunen - sind die Kirchen die größten Arbeitgeber in Deutschland. Sie verfügen über ein hoch differenziertes Instrumentarium der politischen Einflussnahme, etwa durch offizielle Vertreter bei der Bundesregierung und dem Bundestag, entsprechende Vertreter in den Ländern, aber auch ein je eigenes Büro in Brüssel.

Zwar sehen sehr viele Deutsche die Kirchen überaus kritisch, und die Werte in entsprechenden sozialwissenschaftlichen Umfragen lassen erkennen, dass die ganz alten christlichen Sinninstitutionen selbst bei ihren Mitgliedern, den Kir- 
chensteuerzahlen, deutlich und zum Teil dramatisch an Vertrauen eingebüßt haben. Dafür mögen auch diverse Korruptionsskandale in Diakonie und Caritas, Konflikte um pädophile Priester und die mangelnde geistliche Qualität bei zentralen Kasualien, etwa bei Beerdigungen, verantwortlich sein. Auch verlieren die Kirchen weiterhin und neuerdings wieder verstärkt an Mitgliedern; im Jahre 2008 sind 160.000 Protestanten und 135.000 Katholiken aus ihrer Kirche ausgetreten - in nur zwölf Monaten also 295.000 Menschen, aus welchen Gründen auch immer. Aber unübersehbare Krisenphänomene stehen in Kontrast zu vielfältigen Indikatoren für eine bemerkenswerte Stabilität der Kirchen. Das Interesse an christlichen Schulen nimmt gerade bei den bürgerlichen Mittelschichten sehr stark zu, und bestimmte religiöse Angebote, etwa der Gottesdienst am Heiligen Abend, werden von einer kontinuierlich wachsenden Zahl von Glaubenskonsumenten nachgefragt. Wo im öffentlichen Diskurs mehr „Werte“ oder eine „Wiederkehr der Tugend“ eingeklagt wird, hofft man zumeist auf eine Revitalisierung der Kirchen als Moralagenturen, die in unübersichtlicher Krisenzeit verbindliche Orientierung vermitteln sollen. Nicht zuletzt diese hohen Erwartungshaltungen machen die Kirchen zu einem relevanten gesellschaftlichen Akteur mit hohen politischen Einflusschancen. Desto mehr verdient Beachtung, dass die Rolle, die die Kirchen im politischen Betrieb der Bundesrepublik spielen, in der politikwissenschaftlichen Forschung weithin ignoriert worden ist und wird. Auch viele Historiker und speziell Zeithistoriker ignorieren die Kirchen oder lassen erhebliche Schwierigkeiten erkennen, den Ort der Kirchen in der politischen Topographie Deutschlands seit 1945 prägnant zu vermessen. ${ }^{1}$

\section{Die Ankunft der Kirchen im demokratischen Verfassungsstaat}

Beide großen Kirchen im Lande haben sich lange sehr schwer damit getan, normative Prinzipien der modernen parlamentarischen Demokratie zu akzeptieren. Fragen der politischen Ethik wurden schon seit der Französischen Revolution in akademischer Theologie und kirchlichen Öffentlichkeiten intensiv und kontrovers diskutiert. Die große Mehrheit der römisch-katholischen Denker legte sich seit dem frühen 19. Jahrhundert auf eine entschieden antirevolutionäre, restaurative Konzeption politischer Ordnung fest, mit prägnanter Ablehnung klassisch

1 Das zeigt nicht zuletzt der letzte Band von Hans-Ulrich Wehlers großer Gesellschaftsgeschichte: Wehler, H.-U.: Deutsche Gesellschaftsgeschichte, Bd. 5: Bundesrepublik und DDR 1949-1990, München, 2008; dazu kritisch: Graf, F.W.: Glaubensmüder Westen - entchristianisierter Osten?, in: Bahners, P./Cammann, A. (Hg.): Bundesrepublik und DDR. Die Debatte um Hans-Ulrich Wehlers „Deutsche Gesellschaftsgeschichte“, München, 2009, 180-182. 
liberaler Ordnungsvorstellungen. Neothomistisches Naturrechtsdenken wurde seit den 1860er Jahren dann zu einem dezidiert antiliberalen, als dritter Weg zwischen freier Marktwirtschaft und Sozialismus empfohlenen Ordnungsmodell fortentwickelt, das unter dem Leitbegriff „Katholische Soziallehre“ bis in die politischen Debatten der Bundesrepublik hinein starke Prägekraft entfaltete. Mit der Parlamentarisierung der Reichsverfassung im Kaiserreich hatte der effizient organisierte „politische Katholizismus“ allmählich politische Ordnungsideen entwickelt, die ihm nach Weltkrieg und Revolution dann die entschiedene Unterstützung der neuen Republik im Rahmen der „Weimarer Koalition“ mit Sozialdemokraten und Deutscher Demokratischer Partei (DDP) ermöglichten. ${ }^{2}$

Im deutschen Protestantismus war die Diskussionslage komplizierter. Schon in den harten Auseinandersetzungen um die Legitimität der Französischen Revolution und entschiedener politischer Reform bildeten sich hier konkurrierende, einander heftig bekämpfende Fraktionen, die sich, idealtypisch vereinfacht, als bürgerlich-liberaler Kulturprotestantismus einerseits und sozialkonservativer Moralprotestantismus andererseits bezeichnen lassen. Die innerprotestantischen Kulturkämpfe verbanden sich in der Sphäre der Politischen Ethik schon im Vormärz und verstärkt dann im Kaiserreich mit ideenhistorisch spannenden Auseinandersetzungen über das Verhältnis der verschiedenen protestantischen Konfessionskulturen zu modernen liberalen Konzepten von Bürgerfreiheit, Volkssouveränität, Demokratie und Rechtsstaat. Den reformierten, calvinistischen, westlichen Formen des Protestantismus wurde dabei eine spezifische Nähe zu demokratischer Selbstorganisation, Parlamentarismus und entschiedener Bürgerfreiheit zugeschrieben, wohingegen man die überkommenen Ständeethiken des deutschen wie des skandinavischen Luthertums für sozialpaternalistische Gemeinwohlideale bzw. die ideenpolitische Begründung eines starken Sozialstaates verantwortlich machte. In der Tat ließen es die zumeist sehr autoritären Ordnungsethiken im deutschen Luthertum nur eingeschränkt zu, eine vorstaatliche Freiheit des Einzelnen zu denken und die Menschenrechte als normative Grundlage des parlamentarisch-demokratischen Verfassungsstaates anzuerkennen. Im Calvinismus hingegen hatten zentrale Reflexionsmuster der intensiv diskutierten politischen Ethik vergleichsweise früh schon eine hohe Offenheit gegenüber Demokratie, Volkssouveränität und emphatischer Bürgerfreiheit ermöglicht. Zur Überwindung ihrer alten Demokratiefeindschaft und auch eines

2 Als Überblicksdarstellung immer noch unübertroffen: Lönne, K.-E.: Politischer Katholizismus im 19. und 20. Jahrhundert, Frankfurt/M., 1986. 
entschiedenen Antiliberalismus nahmen viele lutherische Ethiker nach 1945 deshalb politische Ordnungskonzepte des westeuropäischen, calvinistischen Protestantismus auf.

Auch im Katholizismus mussten nach dem Ende des Zweiten Weltkrieges und in den Prozessen der Neugründung liberaler Rechtsstaaten überkommene Denkmuster politischer Ethik verabschiedet oder tiefgreifend renoviert werden, um neuen rechtlichen Herausforderungen wie Menschenrechtsindividualismus und der religiös-weltanschaulichen Neutralität des Verfassungsstaates gerecht werden zu können. Die konfliktreichen, intellektuell äußerst spannenden Wege, auf denen der kirchliche Protestantismus in Deutschland wie auch die römischkatholische Kirche, repräsentiert durch die Deutsche Bischofskonferenz, seit den späten 1940er Jahren allmählich demokratiekompatible politische Ethiken entwickelten, können hier nicht im einzelnen nachgezeichnet werden. Denn das diskursive Feld theologischer Ethik ist vielfältig zerklüftet, und selbst die Positionen zentraler theologischer und kirchenpolitischer Akteure sind von den Zeithistorikern noch kaum erforscht worden.

Deutlich ist jedoch: Beide großen Kirchen im Lande haben ihre überkommene Demokratiedistanz in harten, schwierigen Lernprozessen seit den 1950er Jahren zunehmend überwunden, nicht zuletzt dank des entschiedenen Engagements einzelner „Laien“ und Universitätstheologen - exemplarisch genannt seien für den katholischen Diskurs nur Ernst-Wolfgang Böckenförde ${ }^{3}$ und für die protestantischen Kontroversen der Münchner Ethiker Trutz Rendtorff ${ }^{4}$, der als Vorsitzender der „Kammer für öffentliche Ordnung“ der EKD auf dem Höhepunkt der Nachrüstungsdebatte zunächst die sog. Friedensdenkschrift ${ }^{5}$ und dann die sog. Demokratiedenkschrift ${ }^{6}$ entscheidend prägte. Gerade indem die beiden großen Volkskirchen allmählich demokratiekompatible politische Ethiken entwickelten, konnten sie im politischen System der Bundesrepublik Deutschland zu mächtigen staatstragenden Organisationen werden, die insbesondere die sozialpolitischen Debatten über die Ausgestaltung des Sozialstaatsprinzips tiefgreifend geprägt haben. Die beiden Kirchen haben einen erheblichen Anteil an der demokratischen Erfolgsgeschichte der Bundesrepublik. Auch wer die 1989/90 disku-

3 Siehe die gesammelten Studien in: Böckenförde, E.-W.: Recht, Staat, Freiheit. Studien zur Rechtsphilosophie, Staatstheorie und Verfassungsgeschichte, 2. Aufl., Frankfurt/M., 1992.

4 Rendtorff, T.: Vielspältiges. Protestantische Beiträge zur ethischen Kultur, Stuttgart u. a., 1991.

5 Kirchenkanzlei der EKD (Hg.): Frieden wahren, fördern und erneuern, Gütersloh, 1981.

6 Kirchenamt der EKD (Hg.): Evangelische Kirche und freiheitliche Demokratie. Der Staat des Grundgesetzes als Angebot und Aufgabe, Gütersloh, 1985. 
tierte Formel von der ,protestantischen Revolution“ in der DDR ablehnt, ${ }^{7}$ wird der evangelischen Kirche als Organisation und vielen einzelnen Gemeindegliedern und Pfarrern eine entscheidende Rolle in der Delegitimierung des SEDStaats und in der Entwicklung oppositioneller Gegenöffentlichkeiten nicht bestreiten können. Wichtige Gruppen im DDR-Protestantismus können gemeinsam mit nicht-kirchlichen Akteuren, die sich im Schutzraum der evangelischen Kirche vergesellschaften und organisieren konnten, in Anspruch nehmen, in der friedlichen Freiheitsrevolution von 1989 mutig eine Vorreiterrolle übernommen zu haben.

\section{Konfessionskulturelle Unterschiede und diskursive Praxis}

Der Staat des Grundgesetzes hat in diesem Jahr seinen 60. Geburtstag gefeiert. Insoweit scheinen die alten Kontroversen über Christentum, Konfession und Demokratie nur noch für Ideenhistoriker relevant. Aber dies ist genau besehen nicht der Fall. Noch immer kann beim Thema „Kirchen und Christen im demokratischen Verfassungsstaat" von konfessionskulturellen Differenzen zwischen Protestanten und Katholiken sowie von konfessionspolitischem Streit nicht geschwiegen werden. Zwar kann man die viel beschworene „Ökumene“ zwischen den beiden großen Kirchen als einen entscheidenden Faktor für die vergleichsweise hohe Stabilität der alten, Bonner Bundesrepublik würdigen, analog zur Sozialpartnerschaft zwischen den Tarifparteien. Aber trotz aller Ökumene-Rhetorik und gemeinsamer sozialpolitischer Interventionen der beiden großen Kirchen $^{8}$ lassen sich in den leitenden Begriffen der Politischen Ethik und speziell in den normativen Konzepten der Stellung der Kirche in der Welt, d.h., dogmatisch theologisch formuliert, in den jeweils leitenden Ekklesiologien bzw. Kirchentheorien, tiefgreifende Unterschiede beobachten. ${ }^{9}$

7 Zur Diskussion siehe Rendtorff, T.: Protestantische Revolution? Kirche und Theologie in der DDR: Ekklesiologische Voraussetzungen, politischer Kontext, theologische und historische Kriterien, Göttingen, 1993; Kaiser, G./Frie, E. (Hg.): Christen, Staat und Gesellschaft in der DDR. Vorträge und Diskussionen 1993/94, Düsseldorf 1994.

8 Vgl. insbesondere Kirchenamt der Evangelischen Kirche in Deutschland/Sekretariat der Deutschen Bischofskonferenz (Hg.): Für eine Zukunft in Solidarität und Gerechtigkeit. Wort des Rates der Evangelischen Kirche in Deutschland und der Deutschen Bischofskonferenz zur wirtschaftlichen und sozialen Lage in Deutschland, Hannover/Bonn, 1997.

$9 \mathrm{Zu}$ den konfessionsspezifischen Deutungsmustern in der Rechtsethik finden sich wichtige Beiträge in: Dreier, H./Hildendorf, E. (Hg.): Kulturelle Identität als Grund und Grenze des Rechts, Archiv für Rechts- und Sozialphilosophie, Beiheft 113, Stuttgart, 2008. 


\section{Die Rolle des gläubigen Individuums im demokratischen Staat}

Nach den einschlägigen Äußerungen des römischen Lehramtes erwartet „die Kirche" vom katholischen Christen in der politischen Arena, den normativen Vorgaben, Weisungen „der Kirche“ zu folgen. ${ }^{10}$ Die protestantischen Überlieferungen hingegen kennen kein ethisches Mandat der Kirche, das den einzelnen Christen in seinem politischen Handeln binden könnte, auch wenn einzelne und durchaus prominente Theologen im 19. und 20. Jahrhundert immer wieder versucht haben, der Kirche ein ,prophetisches Wächteramt“ gegenüber dem Staat und über die Gesellschaft zuzuschreiben, und vom einzelnen Christen verlangten, sich diese autoritäre Zuordnung von Kirche, Gesellschaft und politischen Institutionen christustreu zu eigen zu machen. Weithin durchgesetzt hat sich im Protestantismus jedoch eine Sicht, die dem einzelnen Christen als Bürger ganz selbstverständlich ein Recht auf Selbständigkeit zuerkennt und seine politische Mündigkeit betont. In protestantischer Perspektive handelt der Christ in der parlamentarischen Demokratie aus eigener politischer Einsicht, und er ist hier nicht an irgendwelche normative Vorgaben kirchlicher Institutionen und Instanzen gebunden. Dies schließt es nicht aus, dass sich die Evangelische Kirche in Deutschland regelmäßig zu Grundfragen politischer Ethik äußert und sich durch Denkschriften am öffentlichen Diskurs über zentrale Probleme des Gemeinwesens beteiligt. ${ }^{11}$

Die EKD will mit solchen Stellungnahmen politischen Akteuren und der interessierten Öffentlichkeit ihre protestantische Sicht zu einem umstrittenen Thema oder gesellschaftlichen Problem erläutern. Auch kann sie dem einzelnen Christen Orientierungen vermitteln und seine Bereitschaft zu politischem Engagement stärken. Aber sie kann und darf nicht erwarten, dass sich der einzelne Christ die in kirchlichen Gremien, etwa in den „Kammern“ der EKD oder von Synoden, erarbeiteten Argumentationen und Sichtweisen in kritikloser Kirchentreue zueigen macht. Folgt er ihnen, dann nur kraft eigener theologischer und politischer Einsicht. Deshalb gilt: Die im folgenden skizzierten Überlegungen spiegeln allein die individuelle Sicht des Autors, eines liberalen protestantischen Theologen, der in seiner Denkarbeit stark geprägt ist von Kantischem Republikanismus,

10 Vgl. Kongregation für die Glaubenslehre: Lehrmäßige Note zu einigen Fragen über den Einsatz und das Verhalten der Katholiken im politischen Leben, 2002.

$11 \mathrm{Zu}$ den verschiedenen Formen öffentlicher Intervention der Evangelischen Kirche in Deutschland siehe nun die neue sog. Denkschriften-Denkschrift Kammer für Öffentliche Verantwortung der EKD: Das rechte Wort zur rechten Zeit. Eine Denkschrift des Rates des Evangelischen Kirche in Deutschland zum Öffentlichkeitsauftrag der Kirche, Gütersloh, 2008. 
freier Theologie des sog. liberalen Kulturprotestantismus und unfanatischem Denkglauben. ${ }^{12}$

In der parlamentarischen Demokratie sind Christen in ganz unterschiedlichen Rollen und Funktionen politisch engagiert: als Wähler, aktive Mitglieder einer politischen Partei, Mandatsträger oder Mitarbeiter in irgendeiner zivilgesellschaftlichen Aktionsgruppe, von Amnesty International über Greenpeace bis hin zu irgendwelchen Naturschützern und Animal-Rights-Aktivisten. Zudem engagieren sich viele Christen ehrenamtlich in ihren Kirchengemeinden oder für die lokalen Sozialtstationen von Gemeindediakonie und Caritas. Oft ist ihr Glaube eine starke motivierende Kraft für ein bürgerschaftliches Handeln, das sich am je gegebenen Ort, im Stadtteil oder in der Gemeinde, um Bewältigung konkreter Not bemüht.

\section{Kirchen als Interessenverbände}

Dieses im weiten Sinne politische Handeln christlicher Bürger ist zu unterscheiden von der gesellschaftlichen Präsenz und politischen Aktion christlicher Organisationen, allen voran der beiden großen Kirchen. Sie nehmen politischen Einfluss auf vielfältigen Wegen wahr: durch klassische Instrumente des Lobbyismus wie der Beeinflussung von Mandatsträgern, durch Öffentlichkeitsarbeit und publizistische Interventionen ihrer leitenden Vertreter, durch regelmäßige Kontaktgespräche mit der Bundesregierung und den Landesregierungen sowie entsprechende Gespräche mit den Vertretern der politischen Parteien, durch die Mobilisierung der kirchlichen Öffentlichkeit in sog. Konsultationsprozessen und nicht zuletzt auch durch die Foren, die der Kirchentag und der Katholikentag den Vertretern der „Amtskirchen“ bieten. Die Kirchen sind mit eigenen Vertretern in den Rundfunkräten der öffentlich-rechtlichen Sender und im Deutschen Ethikrat präsent, und die große Mehrheit der Deutschen erwartet, dass Kirchenvertreter und Theologen in klinischen Ethikkomitees und sonstigen Beratungsorganen für moralische Konfliktfälle präsent sind. Die Kirchen nehmen, bisweilen sehr massiv, Einfluss auf die Besetzung von Enquetekommissionen des Deutschen Bundestags und betreiben in ihren Akademien schon seit den Anfängen der Bundesrepublik sehr intensiv politische Bildungsarbeit - zum Nutzen der Demokratie.

12 Meine Position im vielfältig verminten Spannungsfeld von Religion und Politik habe ich in einem kleinen Essay erläutert: Graf, F.W.: Moses Vermächtnis. Über göttliche und menschliche Gesetze, München, 2006. 
Dabei verfolgen sie, wie jede andere Organisation im pluralistischen Verbändestaat auch, nicht zuletzt organisationsspezifische Interessen: Es geht den kirchlichen Akteuren im politischen Raum immer auch um die Sicherung jenes staatskirchenrechtlich definierten status quo, der unter den Bedingungen der sog. „hinkenden Trennung“ Staat und Kirchen viele Kooperationsmöglichkeiten eröffnet und zugleich den Kirchen staatliche Ressourcen und die Einziehung von Kirchensteuern durch den Staat sichert. Aber das politische Handeln der Kirchen geht im Kampf für die eigenen Interessen und für die Durchsetzung der eigenen ethischen oder politischen Positionen nicht auf. Man muss ihnen zugute halten, dass sie oft Themen kommunizieren oder Probleme aufgreifen, die von anderen gesellschaftlichen Akteuren ignoriert werden. Zudem werden die Kirchen im politischen Betrieb immer wieder mit der Erwartung konfrontiert, die sozialmoralischen Fundamente des demokratischen Gemeinwesens durch Stärkung von Bürgertugend, Solidarität und Nächstenliebe zu festigen und christliche Symbole für eine Art Zivilreligion des neuen deutschen Staates zu aktualisieren. Sie werden hier oft als Werteagenturen wahrgenommen, als intermediäre Institutionen speziell fürs Moralische und Ethische.

Aber die Vertreter der Kirchen sind in all dem, dies ist entscheidend, eben nicht die einzigen und gewiss nicht die wichtigsten Christen in der Demokratie. Jeder Staatsbürger, der zugleich Mitglied einer Kirche oder sonstigen christlichen Gemeinschaft ist, ist Christ in der Demokratie. Und er tut gut daran, genau dies auch in kritischer Distanz zur kirchlichen Organisation, zur sog. „verfassten Kirche“ oder „Amtskirche“, deutlich zu machen.

\section{Neuer Klerikalismus und öffentliche Einflussnahme}

Denn in der politischen Kultur der Bundesrepublik lassen sich mancherlei irritierende Phänomene eines neuen Klerikalismus beobachten. Zum politischen Personal der Berliner Republik gehören nicht nur Berufspolitiker aller möglichen Couleur, sondern auch eine gern moralisierende Klerisei, die zu allem und jedem Stellung nimmt bzw., so ihr Jargon, sich gern „einmischt“. Ein medienbewusster Berliner Bischof kommentiert in der Bild-Zeitung empört ein Gerichtsurteil, so als ob Richterschelte zu seinen genuinen geistlichen Aufgaben gehörte. Ein Augsburger Bischof tritt am ,politischen Aschermittwoch“ bei einer Parteiveranstaltung auf, um die Abtreibungspraxis in der Bundesrepublik als ebenso verwerflich wie die Ermordung von 6 Millionen Juden zu bezeichnen. Und wo es um die Grundrechte von Homosexuellen geht, etwa im Streit um die eheanaloge Legalisierung gleichgeschlechtlicher Partnerschaften oder das Recht auf Adopti- 
on durch gleichgeschlechtliche Paare, zeichnen manche führende Kirchenvertreter Szenarien vom kommenden „Untergang des Abendlandes“ durch die „Zerstörung der Familie“. Die Liste von mancherlei politisch skandalösen öffentlichen Äußerungen hoher kirchlicher ,Würdenträger“ ist lang, und dabei geht es keineswegs nur um die üblichen Verdächtigen in der Deutschen Bischofskonferenz - die Herren Mixa aus Augsburg, Müller aus Regensburg, Meisner in Köln etc. -, sondern auch um zahlreiche Vertreter des evangelikalen Rechtsprotestantismus und Hunderte von Akteuren aus der zweiten und dritten Reihe.

In einer offenen, demokratisch verfassten Gesellschaft hat jeder auch das Recht, die freiheitsgefährdenden Tabus politischer Korrektheit zu durchbrechen. Das Recht auf Meinungsfreiheit gilt auch für Bischöfe, Kirchenfunktionäre und moralisch besonders erregte Fromme. Doch wer im demokratischen Rechtsstaat von der Kanzel herab politisch Partei ergreift, als prominenter Kirchenvertreter den Zustand des Gemeinwesens kritisiert oder demonstrativ sein individuelles Credo verkündet, muss sich seinerseits kritisieren lassen. Ein Bischof hat ja nicht ex officio recht, wenn er in politische Debatten interveniert oder seine Kritik des bösen Zeitgeistes in provokativer Zuspitzung verkündet. Er tut de facto nur, was jeder andere Bürger auch tun darf und sollte: Er nimmt sein Grundrecht auf Meinungsfreiheit wahr, um sich am öffentlichen Streit über gebotene politische Ziele, Wege und Schritte zu beteiligen. Zur Idee des demokratischen politischen Diskurses passt es nicht, wenn einzelne Akteure, beispielsweise Kirchenvertreter, für ihre öffentlichen Sprechakte den Anspruch erheben, man dürfe sie nicht kritisieren.

Offenkundig haben einige prominente kirchliche Sprecher noch immer erhebliche Schwierigkeiten damit, die Spielregeln des freien demokratischen Diskurses zu akzeptieren. Ein neuer klerikaler Autoritarismus lässt sich beobachten: Unterzeichnen einige Theologieprofessoren eine eher maßvolle Petition, in der die Aufhebung der Exkommunikation von politisch rechtsradikalen Bischöfen der „traditionalistischen“ Pius-Bruderschaft kritisiert und das Zweite Vatikanische Konzil als verbindliche Grundlage der Weltkirche beschworen wird, fordert der Regensburger Ortsbischof Gerhard Ludwig Müller öffentlich ein Demütigungsritual ein, mit Entschuldigungsbrief an den Papst, Ablegung eines Treueeides und lautem Sprechen des apostolischen Glaubensbekenntnisses in der bischöflichen Residenz.

Nun kann man argumentieren, dass es hier rein um Innerkirchliches gehe und der Codex Iuris Canonici nun einmal kein Grundrecht des frommen einzelnen, eines jeden „Laien“ auf Meinungsfreiheit auch in der Kirche und gegenüber den ge- 
weihten Klerikern kenne. Begriffe wie Menschenwürde und Menschenrechte sind dem römisch-katholischen Kirchenrecht nun einmal fremd. Anders formuliert: Die diskursive Öffentlichkeit in der Kirche sei per definitionem keine freie Öffentlichkeit im Sinne der modernen bürgerlichen Gesellschaft, und deshalb sei es auch nur konsequent, dass der Vatikanstaat, als einziger europäischer Staat neben Weißrussland, die Europäische Menschenrechtskonvention von 1953 nicht unterzeichnet habe. Auch entspreche es nur der organologischen Ekklesiologie des Zweiten Vatikanischen Konzils und dem konsequent hierarchischen Aufbau der Kirche, dass die „Laien“ und ihre Verbände ihre führenden Vertreter, etwa den Präsidenten des Zentralkomitees der Deutschen Katholiken, zwar wählen können, diese ihr Amt aber nur mit expliziter Zustimmung der Bischöfe dann tatsächlich auch antreten können.

In der Tat gebieten es die modernitätsspezifische Idee der Autonomie der Religion - um 1800 klassisch begründet von Friedrich Daniel Ernst Schleiermacher und die für freiheitliche Gesellschaften konstitutive Idee der strukturellen Differenzierung von Politik und Religion, prägnant zwischen innerkirchlicher Öffentlichkeit und allgemeiner demokratischer Öffentlichkeit zu unterscheiden. Und speziell mit Blick auf die römisch-katholische Kirche ist anzuerkennen, dass es aufgrund der amtstheologischen Grundunterscheidung von Priestern und Laien keine Vorstellung einer prinzipiell gleichen Sachautorität von in der Kirche Sprechenden geben kann; der Bischof spricht ex officio mit ganz anderer Autorität als irgendein Laiengremium, etwa der Diözesanrat. Doch wie sind seine Interventionen in den öffentlichen Diskurs, beispielsweise Stellungnahmen zu biopolitischen Konflikten oder zum Familienrecht, zu beurteilen?

\section{Religiöse Akteure in pluralistischen Diskursen}

Über die Präsenz religiöser Akteure im Diskurs pluralistischer, „offener“ Gesellschaften haben in den letzten Jahren Politische Philosophen spannende Debatten geführt. Der große Liberale John Rawls hat Kriterien für legitime Teilnahme am vernünftigen öffentlichen Diskurs entwickelt. Von den miteinander um beste Lösungen ringenden Bürgern sei insbesondere die Bereitschaft zu erwarten, dem jeweils anderen genau zuzuhören, ihm nicht von vornherein unmoralische Absichten zu unterstellen, sich auf strikt rationale Argumente zu besinnen und pragmatische Kompromissbildung zu fördern. Rawls hat deshalb alle „umfassenden religiösen oder philosophischen Lehren" von vornherein aus dem politischen Diskurs einer freien Bürgergesellschaft auszuschließen verlangt. Denn Vertreter solcher Lehren, beispielsweise Repräsentanten der Amtskirchen, dächten auf- 
grund ihrer Glaubensprämissen und dogmatischen Bindungen strukturell autoritär und seien aufgrund ihres weltanschaulichen Absolutheitsanspruchs weder zu rationaler Verständigung fähig noch zu pragmatischer Konsenssuche bereit. ${ }^{13}$

Man kann, wenn man Voten mancher deutscher Bischöfe hört und liest, für Rawls' Argumentation Verständnis aufbringen. Doch ist es in der parlamentarischen Demokratie freiheitsdienlich, religiösen Sprechern bzw. Vertretern religiöser Organisationen von vornherein das Recht auf Teilnahme am politischen Grundlagenstreit zu verweigern? Jürgen Habermas hat in Fortschreibung seiner Diskursethik ein deutlich liberaleres Modell entwickelt. Religiöse Akteure haben, so Habermas, im öffentlichen Diskurs einer freien Gesellschaft das Recht, ihre Position geltend zu machen, und von entschieden areligiösen, säkularen Bürgern sei zu erwarten, dass sie den Frommen und ihren Vertretern aufmerksam zuhören. ${ }^{14}$ Allerdings sind religiöse Akteure in einer offenen, pluralistischen Gesellschaft nur zivilgesellschaftliche Akteure neben anderen. Müssen die Säkularen aufmerksam den Frommen zuhören, so haben sich diese umgekehrt an bestimmte Grundbedingungen des öffentlichen demokratischen Diskurses zu halten. Sie müssen etwa Spielregeln der Fairness beachten und ihre Sicht rational, in intersubjektiv verständlichen, von anderen nachvollziehbaren Argumenten, kommunizieren. Auch ist von ihnen Respekt vor dem jeweils anderen und die Bereitschaft zur Kompromisssuche zu erwarten.

Viele Stellungnahmen von Vertretern der beiden großen Kirchen im Lande werden diesen elementaren Kriterien des demokratischen Diskurses nicht gerecht. Um nur ein Beispiel zu nennen: In den politischen Konflikten über Sterbebegleitung, Patientenverfügung, selbstbestimmtes Sterben und auch assistierten Suizid, wie sie während der letzten Jahre gerade auch im Deutschen Bundestag intensiv geführt worden sind, hält die Deutsche Bischofskonferenz entschieden am alten, zumeist naturrechtlich begründeten Anspruch der Römisch-Katholischen Kirche fest, der Rechtsstaat müsse in seiner Gesetzgebung den „der Kirche“ erschlossenen Einsichten in die unbedingte „Heiligkeit des Lebens“ folgen. Nun wird im Rechtsstaat kein katholischer Bürger daran gehindert, sein Leben ganz streng nach der Morallehre seiner Kirche zu führen. Aber warum sollten Andersdenkende, gottferne Liberale etwa oder freie Protestanten, von Staats wegen dazu

13 Rawls, J.: The Idea of Public Reason revisited, in: The University of Chicago Law Review, 64/3 (1997), 765-807; zum Kontext: ders.: Political Liberalism, New York, 1993.

14 Habermas, J.: Kognitive Voraussetzungen für den ,öffentlichen Vernunftgebrauch“ religiöser und säkularer Bürger, in: ders.: Zwischen Naturalismus und Religion. Philosophische Aufsätze, Frankfurt/M., 2005, 119-154. 
gezwungen werden dürfen, ihre Lebensmaximen an römisch-katholischer Spezialmoral auszurichten? Der freiheitlich-demokratische Rechtsstaat darf, um seiner freiheitsdienlichen religiös-weltanschaulichen Neutralität willen, nicht die Sondermoral irgendeiner Weltanschauungsgemeinschaft allgemein verbindlich machen wollen. In den Kirchen lassen sich aber immer wieder Tendenzen beobachten, gute Gründe Andersdenkender zu ignorieren und den Staat auf die eigenen Positionen festzulegen. Man redet von der religiös-weltanschaulichen Neutralität des modernen Verfassungsstaates und unterläuft zugleich diese Neutralität, in dem man das Recht an eine metapositive oder vorrechtliche Sittlichkeit an „Werte“ oder das „Sittengesetz“ - zurückbindet.

Zur Aufgabe selbstbewusster Christen in der Demokratie dürfte es deshalb gehören, die Themen Klerikalmacht und politisches Mandat der Kirchen neu auf die öffentliche Agenda zu setzen. Wen genau vertritt ein katholischer Bischof, wenn er die offenen Gesellschaften des Westens pauschal als „Diktaturen des Relativismus" bezeichnet? Für wen spricht eine protestantische Landesbischöfin, wenn sie im Streit um die Sterbegleitung mehr Sensibilität für die je eigene lebensgeschichtliche Lage betroffener Individuen einklagt? Der autoritäre, oft auch besserwisserische Habitus, mit dem manche protestantische wie katholische Bischö$\mathrm{fe}$ in den öffentlichen politischen Streit intervenieren, passt schlecht zu einem demokratischen Diskurs, der, gemäß der gleichen Freiheit aller Bürger, auf eine gemeinsame offene Suche nach besten Lösungen hinausläuft.

Nun mag man im forum internum der Kirchen, in rein kirchlichen Öffentlichkeiten mancherlei episkopale Autoritätseitelkeit ertragen können. Aber im forum externum der demokratischen Öffentlichkeit werden Ansprüche auf Deutungsmacht unausweichlich dem argumentativen Härtetest freier Kritik ausgesetzt. Um des demokratischen Gemeinwesens willen tun freie Christen deshalb gut daran, ihre kirchlichen „Obrigkeiten“ entschieden an die Tugend der Demut und Selbstbegrenzung zu erinnern. Und sie sollten mit aller Entschiedenheit darauf insistieren, dass Kirchenkritik seit spätestens 1517 ein christlich legitimer Ausdruck der „Freiheit eines Christenmenschen“ und seit dem ausgehenden 17. Jahrhundert immer auch als ein integrales Element des Kampfs um Bürgerfreiheit verstanden worden ist. „Deutschlands demokratischer Urzustand ist der Protest vor dem Schloss“, hat der in Berlin lehrende Staatsrechtslehrer Christoph Möllers in seinem wunderschönen kleinen Buch „Demokratie - Zumutungen und Versprechen“ jüngst betont. ${ }^{15}$ 


\section{Kirche und bürgerschaftlicher Protest}

Man muss hinzufügen: Die demokratische Kultur hat sich, nicht zuletzt wegen der antirevolutionären, restaurativen Zweckbündnisse der beiden alteuropäischen Ordnungsmächte Staat und Kirche, in Deutschland immer auch im bürgerschaftlichen Protest gegen ein Kirchentum entwickelt, dessen führende und, jedenfalls im Protestantismus, sehr gern obrigkeitsnahe Vertreter aus der gepredigten Allmacht Gottes starke Macht und Autorität für sich selbst abzuleiten suchten. Grundbegriffe der religiösen Sprache und zentrale Glaubenssymbole, etwa die Vorstellung von der Allmacht des ewigen Schöpfergottes, haben den berufenen Vertretern der christlich-religiösen Institutionen immer auch dazu gedient, gegebene politische Ordnung zu sakralisieren und zugleich den Kirchen eine normative Autorität sui generis zuzuschreiben: Wer viel von der Allmacht Gottes spricht, sieht sich leicht als ihr berufener Mandatar und schreibt sich dann gern selbst Elemente von Omnipotenz zu. Christen tun der Demokratie einen Dienst, wenn sie sich in Sachen „Kirchenpolitik“ engagieren - dies ist ein Neologismus des frühen 19. Jahrhunderts - und in den Kirchen gegen autoritäre Homogenisierung von oben selbst die Pluralität je individueller Glaubenshaltungen stärken.

Christoph Möllers hat in seiner kleinen Demokratiekunde auf die notorische Demokratievergessenheit der Deutschen hingewiesen. „Wir haben demokratische Traditionen, aber wir interessieren uns kaum für sie." ${ }^{16}$ In der Tat ist es um die Erinnerungskultur der parlamentarischen Demokratie in Deutschland schlecht bestellt. Möllers nennt ein schlagendes Beispiel: Nirgends wird in der Bundeshauptstadt Berlin an Hugo Preuß, den Schöpfer der Verfassung der Weimarer Republik, erinnert. Aber aus Steuermitteln ist in Berlin-Mitte ein RosaLuxemburg-Denkmal errichtet worden, ein Gedenkort für eine radikale Kritikerin der parlamentarischen Demokratie.

Solche „demokratische Traditionslosigkeit" ${ }^{\text {“17 }}$ lässt sich auch und besonders deutlich in den Erinnerungskulturen der Kirchen und in den akademischen Theologien beobachten. Gern gedenkt man der Märtyrer des Dritten Reiches. Aber man scheut, in universitärer Geschichtsforschung wie in kirchlicher Geschichtspolitik, die Erinnerung an christliche Theoretiker und Wegbereiter der parlamentarischen Demokratie. Man erklärt Dietrich Bonhoeffer zu einem ,protestantischen Heiligen“, hat aber zu Friedrich Naumann, Martin Rade oder Theodor Heuss nichts 
Kirchliches zu sagen. Man kann die in den Kirchen und leider auch in den akademischen Theologien herrschende Demokratievergessenheit gerade mit Blick auf die Weimarer Republik zeigen: Die theologische Aufmerksamkeit gilt primär den Radikalen, Exaltierten, Republikdistanzierten (oder offenen Gegnern der parlamentarischen Demokratie), nicht aber den liberalen Verteidigern der Republik. Christlich liberale Denktraditionen werden in beiden Kirchen notorisch ignoriert. Und nur selten sind die Funktionäre in den Kirchen zu selbstkritischer Reflexion auf die massiven antidemokratischen Erblasten in den verschiedenen Christentümern bereit.

Man redet von Ökumene und schweigt darüber, dass viele orthodoxe Christentümer, religionssoziologisch gesehen, nur Ethno-Religionen sind, in denen die Nation und ihr Territorium, das „heilige Russland“ etwa, sakralisiert werden, nicht aber das Individuum in seiner vorstaatlichen Würde. Dass in manchen Christentümern Menschenrechte des Einzelnen immer noch abgelehnt oder als Ausdruck falscher, antichristlicher Aufklärung bekämpft werden, verschweigt man im ökumenischen Diskurs sich und anderen; man spricht lieber über Amt und Sakrament oder über Gemeinwohl und Sozialstaat. Gern macht man sich im politischen Tageskampf die normativen Leitbegriffe der Verfassung zueigen, vor allem den in den deutschen Kirchen inzwischen inflationär benutzten Begriff der Menschenwürde. ${ }^{18}$ Nicht selten lässt sich dabei ein imperialistischer Gestus der Begriffsbesetzung beobachten, etwa indem „die Menschenwürde“ zu einer genuin oder gar exklusiv christlichen Idee erklärt und damit eine spezielle kirchliche Deutungsmacht reklamiert wird. Der demokratische Staat gehört jedoch, Gott sei Dank, nicht den Kirchen und die Menschenwürde nicht den Theologen. Christen in der Demokratie mögen sich als Bürger besonderer Art sehen. Aber ihr Glaube garantiert es keineswegs, dass sie besonders gute oder immer schon bessere Demokraten als andere sind.

Moderne liberale Demokratietheorien gehen davon aus, dass am Beginn der Demokratie ein Versprechen wechselseitiger Anerkennung gleicher Freiheit steht. Die parlamentarische Demokratie ist ein - um der Machtbegrenzung und permanenten Herrschaftskontrolle willen institutionell hoch differenzierter freier politischer Verband von Individuen, die kraft autonomer Entscheidung diese bestimmte politische Form gewählt haben. Für die Demokratie ist die Idee

18 Zur inflationären Entwertung der Menschenwürde in den Diskursen beider großer Kirchen siehe meine kritische Analyse: Graf, F.W.: Mißbrauchte Götter. Zum Menschenbilderstreit in der Moderne, München, 2009, bes. $177 \mathrm{ff}$. 
gleicher Freiheit fundamental. Diese Idee bedeutet nicht, dass alle Bürger als Menschen gleich sind, relativiert also nicht Individualität oder vielfältige faktische Unterschiedenheit. Gleiche Freiheit meint vielmehr, dass wir uns wechselseitig als mit einem freien Willen begabte, zu Autonomie befähigte politische Subjekte anerkennen.

Im politischen Diskurs der Moderne seit ca. 1780 ist immer wieder über die Frage gestritten worden, inwieweit sich Vorstellungen der gleichen politischen Freiheit der Bürger auch auf genuin christliche Traditionselemente begründen lassen, etwa die jedem einzelnen Menschen von seinem Schöpfer zuerkannte Gottebenbildlichkeit. Die ideenhistorisch äußerst faszinierenden Debatten über mögliche jüdische und christliche Wurzeln demokratischer Ordnungsentwürfe und Freiheitsvorstellungen haben, über das rein Historische hinaus, immer auch das existentielle Interesse christlicher Politiker und Intellektueller gespiegelt, ihr aktives demokratisches Engagement aus dem eigenen Glauben zu begründen: aktive Bürgertugend als primäre Konkretionsgestalt gelebter Frömmigkeit, oder Gottvertrauen als Kraftquell für politischen Mut und tätige Sorge für das Gemeinwesen. Solche Begründungen mögen für den Frommen (oder eine Gruppe von Gläubigen) individuell hilfreich und plausibel sein, können in der Demokratie aber keinerlei Anspruch auf traditionsstiftende Allgemeingültigkeit erheben: Demokratische Bürger pflegen den in der gleichen Freiheit aller gründenden Regelkonsens, teilen aber keine gemeinschaftlichen Vorstellungen über die ideenpolitischen Ursprünge demokratischer Ordnung.

Auch verweisen die Symbolsprachen der Religion und die normativen Grundbegriffe der parlamentarischen Demokratie in ganz unterschiedliche Imaginationsräume und stehen bleibend in Spannung. Die Demokratie geht aus vom autonomen Bürger, der sich mit anderen freien Bürgern in wechselseitiger Anerkennung gleicher Freiheit zur Organisation politischer Herrschaft zweckrational assoziiert. Die Sprachen der Religion aber haben sehr viel zu tun mit Vorstellungen vom guten Leben in der Herzensbindung an Gott, mit Heil und Verderben, Erlösung und Gemeinschaft der Heiligen. Christen in der Demokratie tun um der Demokratie, aber auch um ihres Glaubens willen gut daran, sich die unaufhebbaren Spannungen zwischen demokratischer Vernunftsprache und religiöser Symbolsprache präsent zu halten. Gern schreiben wir uns im politischen Diskurs der Bundesrepublik die aufgeklärte Fähigkeit zu, zwischen Politik und Religion zu unterscheiden, und wir sprechen anderen, etwa „den Muslimen“ oder bestimmten muslimischen Akteuren diese Differenzierungskompetenz ab. Aber die heilsame Unterscheidung von Religion und Politik ist keine ein für allemal 
erreichte Leistung aufgeklärter Vernunft, sondern muss im politischen Tageskampf und im schnellen religiösen Wandel immer neu begründet und verteidigt werden.

Auch manche christliche Akteure in der Bundesrepublik pflegen eine religiöse Kampfrhetorik, die es kaum erlaubt, zwischen Religion und Politik oder auch zwischen Recht und Moral prägnant zu unterscheiden. Sowohl im Rechtskatholizismus als auch im evangelikalen Protestantismus wird die parlamentarische Demokratie ob ihres Neutralitätsliberalismus nicht selten als „relativistisch“ kritisiert und ein christlicher Werte- oder Sittenstaat beschworen. Selbst der höchste Repräsentant der römisch-katholischen Kirche, Papst Benedikt XVI., hat, als Theologieprofessor Joseph Ratzinger und als Präfekt der römischen Glaubenskongregation, in zahlreichen Texten zur religiösen Lage Europas immer wieder gegen das Mehrheitsprinzip der parlamentarischen Demokratie polemisiert und sein tiefes kulturpessimistisches Leiden an moralischem Pluralismus und liberalistischen Individualismus bekundet. ${ }^{19}$

\section{Schluss}

Das Christliche ist religiös vielfältig, in seinen zentralen Symbolen äußerst spannungsreich und auch in ethischer Hinsicht vielgestaltig. In einem viel diskutierten Vortrag über „Politische Ethik und Christentum“, gehalten beim fünfzehnten Evangelisch-sozialen Kongress, hat der Heidelberger Systematische Theologe Ernst Troeltsch, später einer der führenden liberalen Gelehrtenrepublikaner der Weimarer Republik, im Mai 1904 erklärt: „Das Evangelium enthält überhaupt keine direkten politischen und sozialen Weisungen, sondern ist von Grund aus unpolitisch; es ist nur mit den höchsten Zielen des persönlichen Lebens und der persönlichen Gemeinschaft beschäftigt und nimmt die Verwirklichung dieses Ideals in der Erwartung des baldigen Weltendes und des kommenden Gottesreiches mit einer Energie voraus, neben der die Welt und ihre Interessen überhaupt verschwinden. ${ }^{\prime 20}$

Ihr individueller christlicher Glaube kann von Christen deshalb politisch ganz unterschiedlich konkretisiert werden. Glaubensmotive lassen sich mit höchst

19 Dazu nun zahlreiche Belege in der dezidiert polemischen Streitschrift von Posener, A.: Benedikts Kreuzzug. Der Angriff des Vatikans auf die moderne Gesellschaft, Berlin, 2009. Siehe meine Rezension Graf, F. W.: Das Rad der Geschichte zurückdrehen. Alan Poseners brachiale Polemik gegen Papst Benedikt XVI, in: Neue Zürcher Zeitung, Sonderbeilage Bücherherbst 2009, 12. 10. 2009, 14.

20 Troeltsch, E.: Politische Ethik und Christentum, Göttingen, 1904, 32. 
heterogenen politischen Vorstellungen und Zielen verbinden. Aber der individuelle Glaube markiert zugleich auch eine Grenze zwischen dem Privaten und dem Öffentlichen, die nicht zur Disposition demokratischer Willensbildung steht, auch wenn sie im Einzelnen immer neu bestimmt werden muss. Vielleicht ist das die wichtigste politische Leistung von Christen in der Demokratie: die vielen verschieden glaubenden und lebenden Bürger, die einander allein in gleicher Freiheit, aber eben nicht in irgendwelchen substantiellen Kulturwerten oder gemeinschaftlichen Vorstellungen des Guten verbunden sind, dafür zu sensibilisieren, dass Demokratie eine Herrschaftsform ist, die Unterschiede zulässt, keinen moralischen oder religiösen Vergemeinschaftungszwang kennt und dem Individuum einen großen, aber immer gefährdeten und umkämpften Eigenraum des Privaten lässt.

Das schließt es nicht aus, dass der gemeinsame religiöse Glaube Individuen über äußere, etwa kultische Vergesellschaftung hinaus auch innerlich verbindet, also vergemeinschaftet und darin zwischen Staat und Einzelnem eine „Ligatur“21 bildet. Aber „die Kirchen“ als Organisationen leisten dies nicht, sondern bestenfalls einzelne religiöse Gruppen, Kreise und Netzwerke in ihnen. Beide große Volkskirchen sind zu politisch wie religiös extrem pluralen Organisationen geworden, die die Vielfalt der in ihnen vertretenen Positionen nur noch mit größter Mühe integrieren können. Ein prinzipielles Eigenrecht des Individuums konstituiert eben nicht nur die moderne parlamentarische Demokratie mit ihrer Anerkennung vorstaatlicher Freiheitsrechte des einzelnen, sondern de facto auch die modernen okzidentalen Christentümer - selbst wenn diese religiöse Individualisierung in die offiziösen ekklesiologischen Selbstdeutungen der beiden großen Kirchen bisher kaum Eingang gefunden hat. Anders formuliert: Die Bedeutung der Kirchen als Organisationen gesellschaftlicher Konsensbildung wird weiter schwinden. Sie sind inzwischen weithin damit befasst, ihre massiven internen Konflikte zu pazifizieren, und in genau dem Maße, in dem ihnen dies gelingt, können sie auch als Organisationen sekundärer politischer Integration gedeutet werden.

21 Zum Konzept vgl. Dahrendorf, R.: Das Zerbrechen der Ligaturen und die Utopie der Weltbürgergesellschaft, in: Beck, U./Beck-Gernsheim, E. (Hg.): Riskante Freiheiten. Individualisierung in modernen Gesellschaften, Frankfurt/M., 1994, 421-436. 